\title{
Hypoxia-induced Response in Mitochondria DNA Biogenesis in Retinal Organoids Derived from Pluripotent Stem Cells from Leber Hereditary Optic Neuropathy
}

\section{Kaori Ueda}

Kobe University

Akishi Onishi

RIKEN

Masayo Takahashi

RIKEN

Makoto Nakamura ( $\square$ manakamu@med.kobe-u.ac.jp)

Kobe University

\section{Research Article}

Keywords: hypoxia , mitochondrial DNA (mtDNA), biogenesis, Leber hereditary optic neuropathy (LHON), pluripotent stem cells

Posted Date: February 16th, 2021

DOI: https://doi.org/10.21203/rs.3.rs-197283/v1

License: (c) (1) This work is licensed under a Creative Commons Attribution 4.0 International License.

Read Full License 


\section{Abstract}

This study evaluated the impact of hypoxia on mitochondrial DNA (mtDNA) biogenesis in retinal organoids of patients with Leber hereditary optic neuropathy (LHON) with an mtDNA G11778A mutation. The induced pluripotent stem cells derived from the peripheral blood mononuclear cells of LHON patients and a healthy individual were differentiated into three-dimensional retinal organoids with confirmed morphology and retinal ganglion cell (RGC) markers. The retinal organoids were cultured in alternating hypoxic and normoxic states. The ratio of the mtDNA copy number in an organoid to the mtDNA copy number in the white blood cells was calculated. Under both conditions, the control and LHON organoids had similar morphology and RGC marker patterns. Under the normoxic condition, the LHON organoids had a smaller but similar mtDNA copy number ratio as the controls. Hypoxic exposure significantly reduced the mtDNA copy number ratio in both groups. However, the magnitude of reduction was significantly smaller in LHON organoids than in the controls. One LHON patient had a similar and relative mtDNA copy number in the white blood cells to the controls, but the other patient had a much higher number. Therefore, the hypoxic response of retinal mtDNA biogenesis was reduced in LHON patients.

\section{Introduction}

The human retina, composed of 10 layers, can transmit light stimulation from the external environment to the brain. Retinal ganglion cells (RGCs) play a central role in transmitting visual information. They receive visual stimulations to photoreceptor cells via bipolar cells or amacrine cells and transmit the stimulation to the occipital lobe via the lateral geniculate nucleus. In various types of optic nerve diseases, the damage to the RGCs leads to visual dysfunction.

Mutations in mitochondrial DNA (mtDNA) are presumed to lead to the uncoupling of electron transfer and ATP production in mitochondria, resulting in the excessive production of reactive oxygen species (ROS) and subsequent caspase-independent apoptosis of RGCs.

Leber hereditary optic neuropathy (LHON) is a maternally inherited, acute or subacute, rare intractable optic neuropathy caused by a mitochondrial point mutation. Over $95 \%$ of the LHON patients have point mutation mtG3460A, mtG11778A, or mtT11778C. These mutations, designated as primary mutations, are located within the gene encoding the complex I subunits of oxidative phosphorylation/electron transfer enzyme complexes [1, 2].

LHON is characterized by bilateral visual disturbance and central scotoma [1]. The prevalence of LHON varies between 1 in 31,000 and 113,300 among countries [3-5]. LHON typically attacks teenagers to adults in their thirties. In addition, LHON predominantly affects males, possibly due to the involvement of sex chromosomes [6]; however, the details remain unclear.

LHON differs from other mitochondrial diseases in penetrance or tissue specificity. In contrast to other mitochondrial diseases, LHON exhibits a low penetrance. Only about 50 and $10 \%$ of males and females with the LHON genotype, respectively, develop the LHON phenotype. Unidentified epigenetic or known 
environmental factors, such as a history of smoking or heavy drinking, or both are presumed to trigger the development of LHON in the individuals carrying mtDNA mutants [7]. On the other hand, the most striking feature of LHON is the tissue specificity of its phenotype. Unlike other mitochondrial diseases, in almost all LHON patients, RGCs are almost exclusively affected, leading to optic atrophy. Such a tissue specificity is quite surprising, given that almost all LHON patients possess homoplasmic mtDNA mutations, i.e., all the mtDNA molecules are mutated in the somatic cells.

The copy number of mtDNA also plays a role in many diseases. Mitochondrial DNA copy number varies in cell types due to their different demand for energy; some cells have more than 1000 copies per cell [8]. Cancer, neuronal diseases, smoking, and aging affect the mitochondrial copy number [9-14]. Previous reports indicated that the mtDNA copy number in white blood cells was higher in order of LHONasymptomatic siblings and patients and is the lowest in controls $[15,16]$. In a previous report, the mtDNA copy number and mitochondrial biogenesis, including complex $₫$ activity, of RGCs derived from LHON patients were analyzed [17]. However, the effect of environmental factors on the onset of LHON and the induced pluripotent stem (iPS) cells -derived RGCs has not been investigated.

This study aimed to examine whether environmental stress, such as hypoxia, impacts retinal integrity and mtDNA biogenesis in the retina of LHON patients. First, three-dimensional retinal organoids were generated via the differentiation of the iPS cells derived from LHON patients with the mtG11778A mutation and controls. Somatic cells differentiated from human iPS cells are considered to have similar morphology and functions to those of the original cells. For optic morphogenesis, self-organizing opticcup genesis by a serum-free floating culture of embryoid body-like organoids with quick re-aggregation (SFEBq) method using mouse or human pluripotent stem cells was reported $[18,19]$. In this method, cells expressing RGC markers appeared around one month [20]. Next, the morphology and mtDNA copy number relative to nuclear DNA in LHON-derived retinal organoids under normal and hypoxic conditions were compared to those in the control-derived retinal organoids.

\section{Results}

According to the timeline of the differentiation of iPS cells into three-dimensional (3D) retinal organoids (Fig. 1a), we collected 3D retinas at differentiation day (DD) 35. There was a difference in the size of organoids between the patient-derived organoids and control samples, and the thickness of the retinal layer structure was also different. However, immunostaining revealed no difference in the completion of the retinal layer structure among all the samples. Also, there was no difference in the development of retinal organoid morphology (Fig. 1b, d, f, h) and in the distribution of Crx and Brn3b-positive cells (Fig. 1c, e, g, i) among the LHON patients, the healthy participant, and the control 201B7cells (Fig. 1b-i).

We investigated whether mitochondrial dynamics would change in LHON patients and the healthy participant due to fluctuations in oxygen concentration. We analyzed the kinetics of the mtDNA copy number relative to nDNA in retinal organoids in response to the normoxic and hypoxic conditions (Fig. 2). At the normal condition of $20 \%$ oxygen, control-derived retinal organoids tended to have a higher copy 
number of mtDNA than LHON patient-derived organoids (see data on Day 0 ). The mtDNA copy number of LHON patient-derived organoids was $171 \pm 11$ and $214 \pm 30$, respectively, while the mtDNA copy number of control-derived ones was $281 \pm 15$ and $231 \pm 32$, respectively.

On the other hand, the mtDNA copy numbers in the retinal organoids derived from the control individual and LHON patients were reduced during the first 3 days after switching to the hypoxic condition (Day 0 to 3 in Fig. 2) (Fig. 3). The reduction in mtDNA copy number in retinal organoids derived from LHON patients was significantly lower than those from the healthy individuals $(P=0.002$, ANOVA; $P<0.05$ StudentNewman-Keuls post hoc test).

The relative mtDNA copy number in iPS colonies and peripheral blood cells of these subjects were calculated (Table 1). There was no significant difference between the control- and LHON patient-derived iPS colonies that had been maintained for 7 days. On the other hand, the relative mtDNA copy number in the white blood cells in LHON patient 1 was significantly higher than the control cells, while the number of LHON patient 2 was comparable to the controls. Overall, the mtDNA copy number in the iPS colonies was higher than that in the white blood cells.

Table 1

The mtDNA copy number in peripheral blood cells and iPS cells of the LHON patients.

\begin{tabular}{|llllllll|}
\hline \multicolumn{7}{|l|}{ iPS colonies } & \multicolumn{7}{c|}{ white blood cells } \\
\hline LHON 1 & LHON 2 & $201 \mathrm{~B} 7$ & Control 1 & LHON 1 & LHON 2 & Control 1 & Control \\
& & & & & & & 2 \\
\hline $660 \pm$ & $640 \pm$ & $708 \pm$ & $697 \pm$ & $519 \pm$ & $117 \pm$ & $103 \pm$ & $88 \pm$ \\
7.79 & 5.56 & 7.13 & 13.20 & 5.10 & 1.63 & 1.25 & 2.05 \\
\hline
\end{tabular}

\section{Discussion}

This study has revealed three findings. First, the control and LHON-derived retinal organoids generated by the SFEBq method had similar morphologies and $\mathrm{Crx}$ and Brn3b immunoreactivity patterns. Second, hypoxia rapidly reduced the mtDNA copy number relative to the nuclear DNA in the organoids. The degree of reduction was significantly higher in the control-derived organoids than in the LHON patient-derived ones. Lastly, although the mtDNA copy number in white blood cells was significantly higher in a LHON patient with systematic diseases than the controls, it was comparable in a LHON patient without systemic diseases and the controls. There was no difference in the mtDNA copy number in the iPS cell colonies and retinal organoids derived from the two LHON patients.

The mtDNA copy number is directly linked to energy production. It was previously reported that the mtDNA copy number in peripheral blood cells was highest in LHON carriers, who had an mtDNA point mutation and did not develop LHON phenotypes, followed by LHON patients and controls [16, 25]. Therefore, in mitochondria with mild to moderate dysfunction in electron transfer, which may occur in LHON carriers, mtDNA biogenesis may be increased to compensate for the impaired energy production. 
However, if the electron transfer complex has severely deteriorated, which is presumably the case in symptomatic LHON patients, the excessive electrons would increase ROS production, resulting in ROSinduced RGC apoptosis.

It has been reported that anaerobic metabolism by hypoxia, rather than aerobic metabolism, is effective and beneficial for tissue growth in a mouse model of mitochondrial disease [26]. In steady-state energy production, mitochondria efficiently produce large amounts of ATP via aerobic metabolism. In contrast, a diseased condition, such as a mouse model of Leigh's encephalomyelopathy, grows better under hypoxia than the normal oxygen concentration [26]. This observation may be because, under the hypoxic condition, the diseased cells preferentially utilize glycolysis, which is not impaired, more than the impaired electron transport complex; thus, the ROS production and eventual cell death are reduced.

Therefore, reduced mtDNA biogenesis under hypoxia in retinal organoids is likely an adaptive response to less utility of oxygen during energy production. The decreased mtDNA biogenesis in LHON patient-derived retinal organoids under hypoxia may indicate that mtDNA 11778 mutants have an impaired adaptive response to hypoxia. Interestingly, the relative mtDNA copy number in the iPS colonies was similar between the controls and LHON patients; this may be one reason why LHON exclusively affects RGCs.

The mtDNA copy number was higher in the peripheral blood cells of LHON patient 2, who had systemic diseases and was considered a "LHON plus," than those in LHON patient 1, who did not have systemic diseases, and the controls. The number of mutated mtDNA molecules in white blood cells in LHON patient 2 may be associated with the patient's systemic diseases, consistent with the previous reports [16, 25].

It is generally explained that differentiated cells increase their mtDNA copy numbers and produce more energy than undifferentiated iPS cells [27]. Contrary to previous reports, we observed that the mtDNA copy number in retinal tissue differentiated from iPS cells was lower than the undifferentiated iPS colonies. The cause of this phenomenon remains unclear.

Here, we only examined the mtDNA copy number in two patients. More samples will be required to elucidate whether the mtDNA biogenesis or kinetics is associated with the development of the LHON phenotype in individuals carrying the corresponding mtDNA mutations and whether hypoxia indeed triggers the development of LHON. In addition, since we calculated the mtDNA copy number of the entire retinal tissue, it is unclear whether the changes in mIDNA copy numbers occurred specifically in RGCs. Therefore, it is necessary to measure the mtDNA copy number and its kinetics under normoxic and hypoxic cycles in purified RGCs to investigate whether hypoxia or other stress impacts the mtDNA biogenesis and survival of RGCs in LHON patients.

\section{Methods}

\section{Establishment of iPS cells}


This study followed the tenets of the Declaration of Helsinki and was approved by the Institutional Ethics Committee of RIKEN Center for Biosystems Dynamics Research (authorization number: KOBE-IRB-13-23) and Kobe University (authorization number: 1624). Two LHON patients ("LHON 1" and "LHON 2") with the mtDNA G11778A mutation and a healthy volunteer (Control 1) participated in the study. LHON 2 also has psychiatric disorder and type 2 diabetes mellitus and is thus deemed to have LHON plus, i.e., a LHON patient with systemic diseases [22].

LHON 1, LHON 2, and Control 1 each provided $40 \mathrm{ml}$ of peripheral blood in Kobe University Hospital. Each iPS line was established by delivering episomal vectors (Center for iPS Cell Research and Application, Kyoto University) into peripheral blood mononuclear cells isolated from whole blood via electroporation. Patient-derived iPS cells were established from LHON 1 and LHON 2's samples, as previously reported [21]. Control iPS cells were derived from Control 1's sample using the same method. Another control sample (Control 2) was also established from PBMCs (iPS Portal, Kyoto, Japan) as previously described [23]. The LHON 1 iPS cells were deposited to RIKEN Bioresource Center (HPS1900, https://cellbank.brc.riken.jp/cell_bank/CellInfo/?cellNo=HPS1900). Lastly, the 201B7 cells were obtained (Center for iPS Cell Research and Application, Kyoto University).

\section{Differentiation of three-dimensional retinal organoids}

The iPSCs in this study were maintained and differentiated into 3D retinal organoids as described [19, 24]. Briefly, 3D retinal organoids were differentiated from iPS cells and maintained for 6 or 7 days. The iPS cells were dissociated and disseminated onto 96-well V-bottom plates at a density of 12,000 cells/well (Sumitomo Bakelite, Tokyo, Japan) in a differentiation medium (1:1 mixture of Iscove Modified Dulbecco Medium and F12 medium) (Gibco) supplemented with $10 \%$ knockout serum replacement (KSR), 1-thioglycerol, and lipid emulsion. On DD 6 or 7, 1.5 nM human BMP4 (R\&D Systems, Minneapolis, MN, USA) were added to each well. Every 3 days, half the medium was replaced. On DD18 or 19, retinal organoids were harvested and removed to a 90-mm low-cell binding dish (Sumitomo Bakelite, Tokyo, Japan) in Dulbecco's Modified Eagle Medium: Nutrient Mixture F-12 (DMEM/F12; Life Technologies) supplemented with $1 \%$ of $\mathrm{N} 2$ supplement (GIBCO), VEGFR/FGFR inhibitor (SU5402; Sigma-Aldrich Corp.), and 3 mM GSK-3 inhibitor (CHIR99021; Stemgent, Cambridge, MA, USA). On DD21, the medium was changed to DMEM/F12 supplemented with $10 \%$ fetal bovine serum, $1 \%$ N2 supplement, $100 \mathrm{nM}$ retinoic acid (Sigma-Aldrich Corp), and $50 \mu \mathrm{M}$ taurine (Sigma-Aldrich Corp).

\section{Immunohistochemistry}

The 3D-retinal organoids were fixed with 3\% paraformaldehyde/phosphate-buffered saline (PFA/PBS) with $7.5 \%$ sucrose for $60 \mathrm{~min}$, washed in $7.5 \%$ sucrose/PBS for $15 \mathrm{~min}$, cryoprotected in $15 \%$ sucrose, and then in $30 \%$ sucrose, embedded with an OCT compound (Sakura Finetek, Tokyo, Japan), and cryopreserved. 
Frozen sections with a thickness of $10 \mu \mathrm{m}$ were heated in retrieval solution ( $0.1 \mathrm{M}$ citric acid, pH6.0) to improve antibody detection efficiency. After $1.5 \mathrm{~h}$ of blocking in $5 \%$ horse serum in $0.1 \%$ triton-X-100/PBS, the sections were incubated with the primary antibody against Crx (Takara Bio, 1:500) or Brn3b (Santa Cruz Biotechnology, 1:500) in 1\% horse serum overnight, and then incubated with secondary antibodies Alexa Fluor 488 or 546, respectively. Next, the sections were counterstained with 4'6-diamidino-2phenylindole (DAPI) and imaged using a confocal microscope (LSM700; Carl Zeiss, Japan, Germany).

\section{Exposing retinal organoids to hypoxia}

The retinal organoids harvested on around DD35 were, on designated Day 0 , exposed to low oxygen concentration at $5 \%$ for 3 days, and then returned to normal oxygen concentration at $20 \%$. The session of hypoxic exposure was repeated three times.

\section{Measurement of the relative mtDNA copy number}

On days $0,3,6,9,12,15$, and 18,3 retinal organoids derived from each research participant were collected. Whole-genome DNA was extracted using a DNeasy Blood and Tissue Kit (Qiagen) according to the manufacture's protocol. The extracted whole-genome DNA was quantified, and its purity was evaluated by the A260/280 absorbance ratios using an ultraviolet spectrometer (NanoDrop, Thermo Fisher Scientific, Massachusetts, USA).

An mtDNA copy number relative to nuclear DNA (nDNA) was measured using real-time PCR with a human mtDNA detection primer set (Human Mitochondrial DNA (mtDNA) Monitoring Primer Set, Takara Bio Inc., Kusatsu, Japan). Briefly, the extracted genomic DNA was mixed with four primer pairs, including mtDNA primer pairs ND1 and ND5 and nDNA primer pairs SLCO2B1 and SERPINA1, to detect mtDNA and nDNA. PCR was performed at $95^{\circ} \mathrm{C}$ for $30 \mathrm{sec}, 95^{\circ} \mathrm{C}$ for $5 \mathrm{sec}$ for 40 cycles, and at $60^{\circ} \mathrm{C}$ for $30 \mathrm{sec}$. The difference between the $\mathrm{Ct}$ values of the ND1 and SLCO2B1 reactions was defined as $\triangle \mathrm{Ct} 1$, and the difference between the $\mathrm{Ct}$ values of the ND5 and SERPINA1 reactions was defined as $\triangle \mathrm{Ct} 2$. MtDNA copy number was calculated as the average of $2^{\Delta \mathrm{Ct} 1}$ and $2^{\Delta \mathrm{Ct} 2}$.

\section{Statistical Analysis}

All statistical analysis were performed by Med Calc (version 19.6.4; MedCalc Software, Mariakerte, Belgium) that statistical significance was set at $P<0.05$.

\section{Declarations}

\section{Ethics declarations}


This study followed the tenets of the Declaration of Helsinki. It was approved by the Institutional Ethics Committee of RIKEN Center for Biosystems Dynamics Research (authorization number: KOBE-IRB-13-23) and Kobe University (authorization number: 1624). All experiments were performed in accordance with. Informed consent was obtained from all participants.

\section{Data Availability}

The data related to this study will be available from the corresponding author, Makoto Nakamura, upon reasonable request.

\section{Acknowledgements}

We are grateful to Michiru Matsumura, Kazuko Tsujimoto, Yukako Hirao, Shoko Fujino, Kanako Kawai, Kyoko Iseki, Tomoyo Hashiguchi for technical support, and all members of the M.T. laboratory and Keiko Muguruma for discussions. We are also grateful to Megumi Kitamura and Keiko Kojima for technical support and all members of the M.N. laboratory for discussions. This study was supported in part by grants from the Research Center Network for Realization of Regenerative Medicine (MEXT), the Program for Intractable Disease Research Utilizing Disease-Specific iPS Cells (JST and AMED).

\section{Author Contributions}

K.U. designed the study, performed the experiments, analyzed the data, and wrote the manuscript. A.O. supervised the experiments and analyzed the data. M.T. supervised the project. M.N. designed the study, supervised the project, analyzed the data, and wrote the manuscript.

\section{Competing Interest Statement}

The authors declare no competing interests.

\section{References}

1. Meyerson, C., Van Stavern, G. \& McClelland, C. Leber hereditary optic neuropathy: current perspectives. Clinical ophthalmology (Auckland, N.Z.). 9, 1165-1176 (2015).

2. Mackey, D. A. et al. Primary pathogenic mtDNA mutations in multigeneration pedigrees with Leber hereditary optic neuropathy. Am. J. Hum. Genet. 59, 481-485 (1996).

3. Man, P. Y. W. et al. The epidemiology of Leber hereditary optic neuropathy in the North East of England. Am. J. Hum. Genet. 72, 333-339 (2003). 
4. Puomila, A. et al. Epidemiology and penetrance of Leber hereditary optic neuropathy in Finland. Eur. J. Hum. Genet. 15, 1079-1089 (2007).

5. Ueda, K. et al. Nationwide epidemiological survey of Leber hereditary optic neuropathy in Japan. J. Epidemiol. 27, 447-450 (2017).

6. Nakamura, M., Fujiwara, Y. \& Yamamoto, M. The two locus control of Leber hereditary optic neuropathy and a high penetrance in Japanese pedigrees. Hum. Genet. 91, 339-341 (1993).

7. Kirkman, M. A. et al. Gene-environment interactions in Leber hereditary optic neuropathy. Brain. 132, 2317-2326 (2009).

8. Robin, E. D., Wong, R. \& Mitochondrial DNA molecules and virtual number of mitochondria per cell in mammalian cells. J. Cell. Physiol. 136, 507-513 (1988).

9. Chen, S. et al. Elevated mitochondrial DNA copy number in peripheral blood cells is associated with childhood autism. BMC Psychiatry. 15, 50 (2015).

10. Rice, A. C. et al. Mitochondrial DNA copy numbers in pyramidal neurons are decreased and mitochondrial biogenesis transcriptome signaling is disrupted in Alzheimer's disease hippocampi. J. Alzheimers Dis. 40, 319-330 (2014).

11. Giordano, L. et al. Cigarette toxicity triggers Leber's hereditary optic neuropathy by affecting mtDNA copy number, oxidative phosphorylation and ROS detoxification pathways. Cell Death. Dis. 6, e2021 (2015).

12. Mengel-From, J. et al. Mitochondrial DNA copy number in peripheral blood cells declines with age and is associated with general health among elderly. Hum. Genet. 133, 1149-1159 (2014).

13. Thyagarajan, B., Wang, R., Barcelo, H., Koh, W. P. \& Yuan, J. M. Mitochondrial copy number is associated with colorectal cancer risk. Cancer Epidemiol. Biomarkers. Prev. 21, 1574-1581 (2012).

14. Masayesva, B. G. et al. Mitochondrial DNA content increase in response to cigarette smoking. Cancer Epidemiol. Biomarkers. Prev. 15, 19-24 (2006).

15. Bianco, A. et al. Mitochondrial DNA copy number differentiates the Leber's hereditary optic neuropathy affected individuals from the unaffected mutation carriers. Brain. 139, e1 (2016).

16. Bianco, A. et al. High mitochondrial DNA copy number is a protective factor from vision loss in heteroplasmic Leber's hereditary optic neuropathy (LHON). Invest. Ophthalmol. Vis. Sci. 58, 21932197 (2017).

17. Wu, Y. R. et al. Bioactivity and gene expression profiles of hiPSC-generated retinal ganglion cells in MT-ND4 mutated Leber's hereditary optic neuropathy. Exp. Cell Res. 363, 299-309 (2018).

18. Eiraku, M. et al. Self-organizing optic-cup morphogenesis in three-dimensional culture. Nature. $\mathbf{4 7 2}$, 51-56 (2011).

19. Nakano, T. et al. Self-formation of optic cups and storable stratified neural retina from human ESCs. Cell Stem Cell. 10, 771-785 (2012).

20. Kobayashi, W. et al. Culture systems of dissociated mouse and human pluripotent stem cell-derived retinal ganglion cells purified by two-step immunopanning. Invest. Ophthalmol. Vis. Sci. 59, 776-787 
(2018).

21. Wu, Y. R. et al. Bioactivity and gene expression profiles of hiPSC-generated retinal ganglion cells in MT-ND4 mutated Leber's hereditary optic neuropathy. Exp. Cell Res. 363, 299-309 (2018).

22. Okita, K. et al. A more efficient method to generate integration-free human iPS cells. Nature Methods. 8, 409-412 (2011).

23. Finsterer, J. \& Zarrouk-Mahjoub, S. Leber's hereditary optic neuropathy is multiorgan not mono-organ. Clin. Ophthalmol. (Auckland, N.Z.). 10, 2187-2190 (2016).

24. Edo, A. et al. Capacity of retinal ganglion cells derived from human induced pluripotent stem cells to suppress T-cells.Int. J. Mol. Sci.21 (2020).

25. Kuwahara, A. et al. Generation of a ciliary margin-like stem cell niche from self-organizing human retinal tissue. Nat. Commun. 6, 6286 (2015).

26. Giordano, C. et al. Efficient mitochondrial biogenesis drives incomplete penetrance in Leber's hereditary optic neuropathy. Brain. 137, 335-353 (2014).

27. Jain, I. H. et al. Hypoxia as a therapy for mitochondrial disease. Science (New York, N.Y.). 352, 54-61 (2016).

28. Xu, X. et al. Mitochondrial regulation in pluripotent stem cells. Cell Metab. 18, 325-332 (2013).

\section{Figures}

(a)

(Differentiation Day)

\begin{tabular}{ll}
0 & 6 \\
\hline $\begin{array}{l}1.2 \times 10^{4} / \text { well } \\
96 \text { well }\end{array}$ & $\uparrow$ IMDM / F-12, $10 \%$ KSR \\
BMP4
\end{tabular}
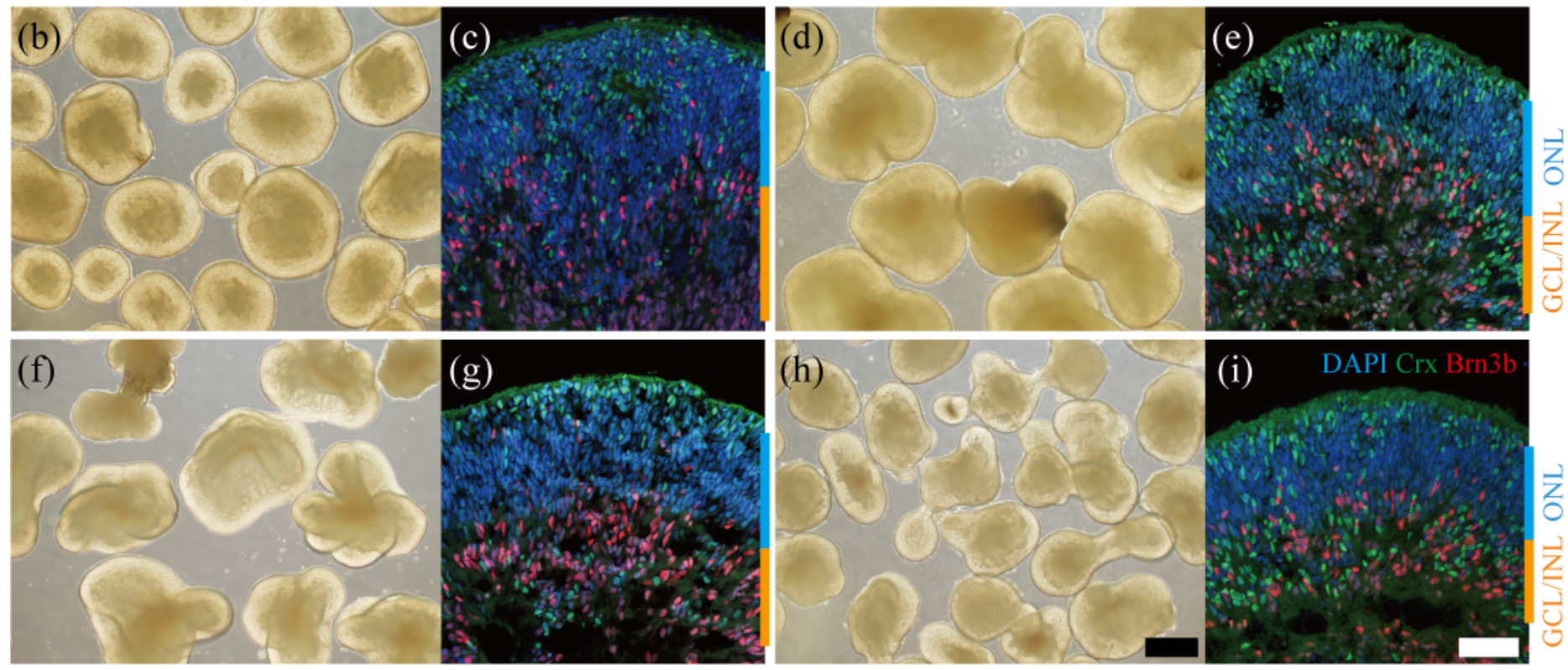
Figure 1

Three-dimensional (3D) retinal organoid derived from LHON / control iPS cells. a. The Schema of the differentiation protocol of LHON and control iPS cells. b-i. Microscopic images of retinal organoids differentiated from LHON patient 1 (b), patient 2 (d), and Control 1 (h) and 201 B7 cells (f) of differentiation day around 40 . Sections of 3D retinal organoids derived from LHON patient 1 (c), patient 2(e), 201B7(g), Control 1(i). Scale bars: $500 \mu \mathrm{m}$ (black, b, d, f, h), $50 \mu \mathrm{M}$ (c, e, g, i).

(Copies)

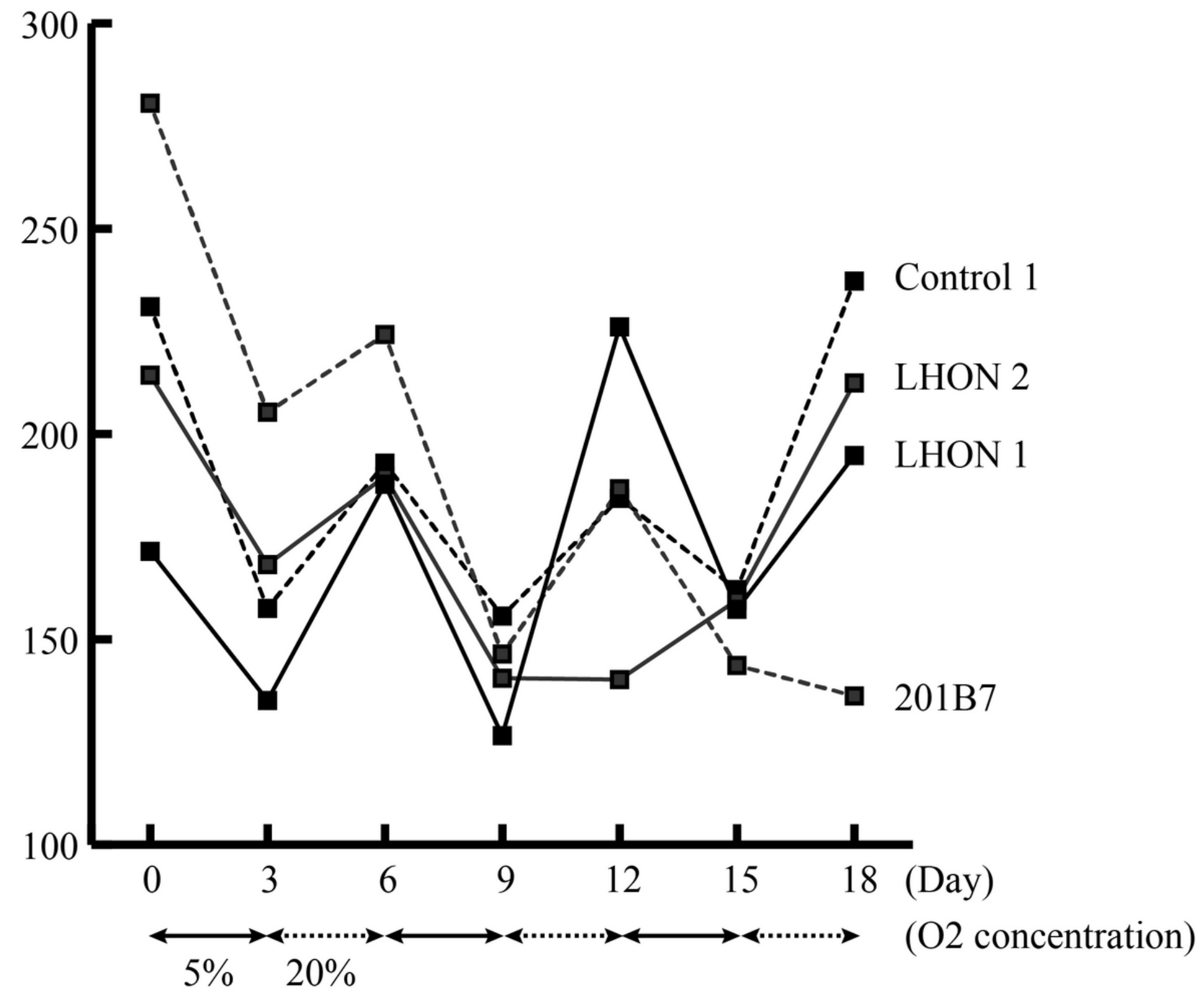

Figure 2

Variation of mtDNA copy number in three-dimensional retinal organoid derived from LHON patients and control iPS cells. 
(Change in mtDNA copy number)

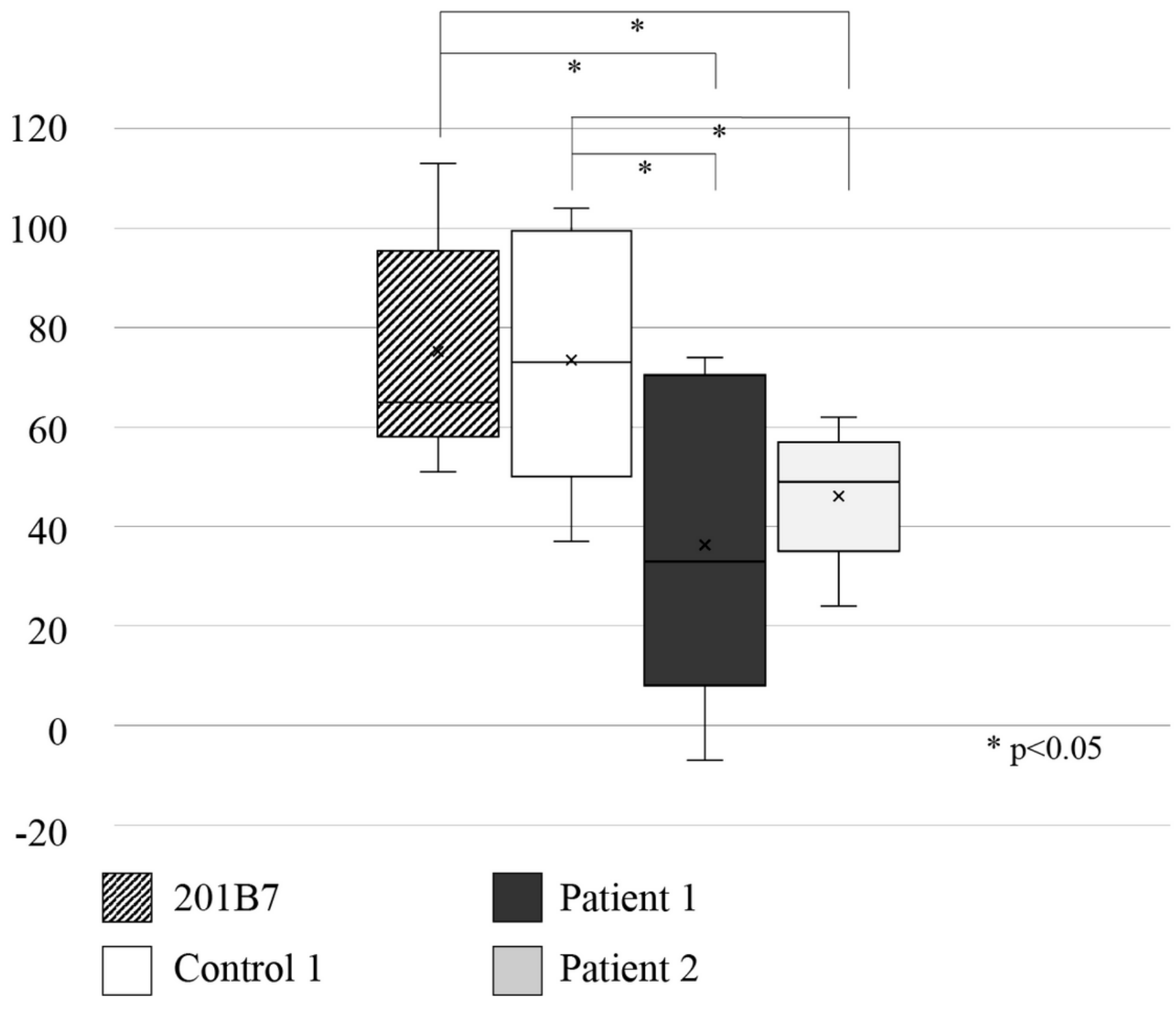

Figure 3

Decrease in mtDNA copy number when oxygen concentration is reduced from $20 \%$ to $5 \%$ on Day $0-3$ in Figure 2. 